\title{
Un mot de la rédactrice
}

La diversité, la vitalité et l'adaptabilité de notre communauté dévaluation sont à l'honneur dans le présent numéro de la RCEP. Je suis heureuse de vous présenter une nouvelle section, intitulée Roots and Relations: Celebrating Good Medicine in Indigenous Evaluation (« Nos racines et nos liens: Célébrons lévaluation autochtone »). Larry Bremner et Nicole Bowman, qui ont gracieusement accepté d'agir à titre de rédacteur et rédactrice de section, nous donnent une vue d'ensemble de leur vision pour la section, qui vise à donner loccasion aux étudiantes et étudiants, aux chercheuses et chercheurs, aux praticiennes et praticiens, aux responsables des politiques et aux évaluatrices et évaluateurs autochtones de nous faire part de leurs travaux de différentes façons. Il s’agit d'une étape importante de la décolonisation de nos pratiques dévaluation et de recherche, et toute notre équipe est très reconnaissante à légard de ceux et celles qui contribueront à cet espace dans les années à venir.

Ensuite, un segment thématique, supervisé par Marlène Laeubli Loud, tire des leçons de lévaluation en temps de pandémie et traite des façons dont les approches et les processus doivent s'adapter à des changements sociaux et environnementaux inédits. Le segment s'enrichit de contributions de Zenda Ofir sur le rôle de la coopération sud-sud dans lévolution de narratifs traditionnels en évaluation; de Louise Gallagher et de Zenda Ofir sur les droits de la nature comme cadre conceptuel pour la pratique dévaluation; et d'Adam Hejnowicz et de Scott Chaplowe sur la science des données et son potentiel relatif à lévaluation. Ces sujets seront certainement d'un grand intérêt pour les évaluatrices et les évaluateurs de partout dans le monde.

Je suis aussi heureuse de présenter quatre autres contributions dans le cadre de ce numéro : le premier, par Jori Hall, traite de compétences culturelles comme élément essentiel de la pratique d'évaluation et propose un nouveau cadre de travail fondé sur les travaux du philosophe Emmanuel Levinas. Le lien entre cet article et les deux sections précédentes est puissant et nous pousse à réfléchir sur la façon de développer notre propre compétence culturelle dans le cadre de notre pratique. Ensuite vient un article de Kristina Kopps (travaillant avec des collègues, des aînés et des gardiennes et gardiens du savoir) qui présente un cadre dévaluation de programme autochtone. Une note sur la pratique de Chris Voegli sur les groupes consultatifs en matière dévaluation nous offre des lignes directrices utiles sur la façon de structurer la participation de parties prenantes, et se fonde sur une synthèse sur la la littérature et de pratiques actuelles. Et finalement, une note sur la pratique de Janice Duddy vient mettre en relief un exemple dévaluation de l'impact sur la participation de programmes de leadership pour des personnes atteintes du VIH.

Finalement, deux comptes rendus de livres viennent compléter le numéro pour nous donner des suggestions de lecture et des conseils. 
Je remercie du fond du cœur toutes les contributrices et les contributeurs qui continuent à nous enseigner de nouvelles choses, à nous poser des défis et à nous aider à nous adapter à de nouvelles façons de réfléchir et de pratiquer lévaluation.

Isabelle Bourgeois, Ph. D. Rédactrice Revue canadienne d'évaluation de programme 